\title{
Comparison of Multifactor Vs Independent Marker in Predicting Severity of Acute Pancreatitis
}

\author{
Vijaykumar C Bada ${ }^{\circledR}$ \\ Head, Department of Surgical gastroenterology \& Robotic Sciences, KIMS Hospitals, Kondapur. Hyderabad, Telangana, India.
}

\section{Abstract}

Background: Acute pancreatitis (AP) is an inflammatory process with a highly variable clinical course. This study was conducted to compare the multifactor vs independent marker in predicting the severity of acute pancreatitis. Subjects and Methods: The present study comprised of 50 patients of acute pancreatitis. In all patients, reactive protein (CRP), Interleukin-6 (IL-6), PMN-Elastase (PMN-E), Procalcitonin (PCT), RANSON"s score, GLASGOW score, APACHE-II score, APACHE-O score and Balthazar's CTSI score was recorded. Results: There were 45 males and 5 females in the study. There were 12 (22.64\%) obese patients in this study. The age of the patients was a significant indicator to discriminate or predict patients with mild or severe pancreatitis. With an AUROC of 0.6004 , it was found that age was a poor predictor of the severity of acute pancreatitis. Obesity of the patients was a significant indicator to discriminate or predict patients with mild or severe pancreatitis. With an AUROC of 0.6004, it was found that age is a poor predictor of the severity of acute pancreatitis. Organ failure at admission is more likely to reflect severe cases, it is found to be a poor predictor of severity in acute pancreatitis. The mean CTSI score in the study was 3.57 (SD 2.64), with a median of 2 and ranged from 1 to 10 . It was higher in severe pancreatitis and a CTSI score of $>=3$ was significantly associated with patients with acute pancreatitis by bivariate analysis. Conclusion: The authors found that overall, CRP was the best predictor, followed by IL-6, CTSI score, PCT, Glasgow, Ranson's and APACHE-II. PMN-Elastase, Age, obesity and organ failure at admission are poor predictors of severity of acute pancreatitis.

Keywords: Acute Pancreatitis, APACHE-II, Scoring systems

Corresponding Author: Vijaykumar C Bada, Head, Department of Surgical gastroenterology \& Robotic Sciences, KIMS Hospitals, Kondapur. Hyderabad, Telangana, India.

E-mail: vijaysge@gmail.com

Received: 15 April 2020

Revised: 29 May 2020

Accepted: 11 June 2020

Published: 30 June 2020

\section{Introduction}

Acute pancreatitis (AP) is an inflammatory process with a highly variable clinical course. ${ }^{[1]}$ Most patients with AP have a mild disease that resolves spontaneously without sequelae. However, $10 \%-20 \%$ of patients experience a severe attack with high mortality of up to $30 \% .{ }^{[2]}$ This high-risk group of patients may benefit from aggressive fluid resuscitation, close monitoring for the development of organ failure, proper administration of antibiotics and specific therapeutic procedures, such as endoscopic sphincterotomy and radiologic intervention. Therefore, early assessment of the severity and identification of patients at risk is essential for first intensive therapy and timely response and has been shown to improve prognosis and survival. ${ }^{[3]}$

Most patients with acute pancreatitis have a mild form of the disease that will respond to supportive treatment. Approximately $20 \%$ of affected individuals will develop a severe clinical course in association with the development of a systemic inflammatory response syndrome (SIRS), multiple organ failure (MOF), and on occasion, death. Severe attacks of pancreatitis are associated with prolonged hospitalization, significant morbidity, and mortality ranging between $30 \%$ and $50 \% .{ }^{[4]}$

The AP classification criteria established by the 1992 Atlanta International Symposium used Acute Physiology and Chronic Health Evaluation (APACHE) II scores $\geq 8$ and Ranson scores $\geq 3$ as early markers of SAP. Subsequently, many guidelines have also recommended using APACHE II and Ranson scores to assess disease severity at $24-48 \mathrm{~h}$ after admission. ${ }^{[5]}$ Moreover, specific instructions recommend using computed tomography severity index (CTSI) scores $\geq 3$, C-reactive protein (CRP) levels $\geq 150 \mathrm{mg} / \mathrm{L}$ and hematocrit (HCT) levels $\geq 44$ to predict SAP. Recently, procalcitonin (PCT), Cr and blood urea nitrogen (BUN) have been used to predict SAP and mortality. ${ }^{[6]}$ This study was conducted to compare the 
multifactor vs independent marker in predicting the severity of acute pancreatitis.

\section{Subjects and Methods}

The present study was conducted in the Department of Surgical Gastroenterology. It consisted of comprised of 50 patients of acute pancreatitis of both genders. All patients were informed regarding the study and their written consent was obtained. Ethical clearance was obtained from the institutional ethical committee.

Inclusion criteria were all patients who present with acute pancreatitis with the above diagnostic tests and patients who came within 72 hours of the onset of symptoms. Exclusion criteria were all patients who came more than 72 hours after the start of symptoms.

Data such as name, age, gender etc. were recorded. A detailed general and systemic examination were done in all patients. All the patients were subjected to complete blood counts (CBC), S. electrolytes, chest X-ray, USG, contrast-enhanced CT Scan abdomen, C-reactive protein (CRP), Interleukin-6 (IL-6), PMN-Elastase (PMN-E), Procalcitonin (PCT). In all patients, RANSON"s score, GLASGOW score, APACHE-II score, APACHE-O score and Balthazar's CTSI score were recorded. Results thus obtained were subjected to statistical analysis. A P-value of less than 0.05 was considered significant.

Results

\begin{tabular}{|lll|}
\hline \multicolumn{2}{l|}{ Table 1: Sex distribution } \\
\hline Total- 50 & \\
\hline Gender & Male & Female \\
\hline Number & 45 & 5 \\
\hline
\end{tabular}

[Table 1] shows that there were 45 males and 5 females in the study.

\begin{tabular}{|lll|}
\hline \multicolumn{2}{|l|}{ Table 2: Obesity distribution } & \\
\hline Obesity & Number & Percentage \\
\hline Obese & 12 & 22.64 \\
Non-Obese & 41 & 77.36 \\
Total & 53 & 100 \\
\hline
\end{tabular}

[Table 2] shows that there were 12 (22.64\%) obese patients in this study.

[Figure 1] shows that the age of the patients was a significant indicator to discriminate or predict patients with mild or severe pancreatitis. With an AUROC (Area Under ROC) of 0.6004,

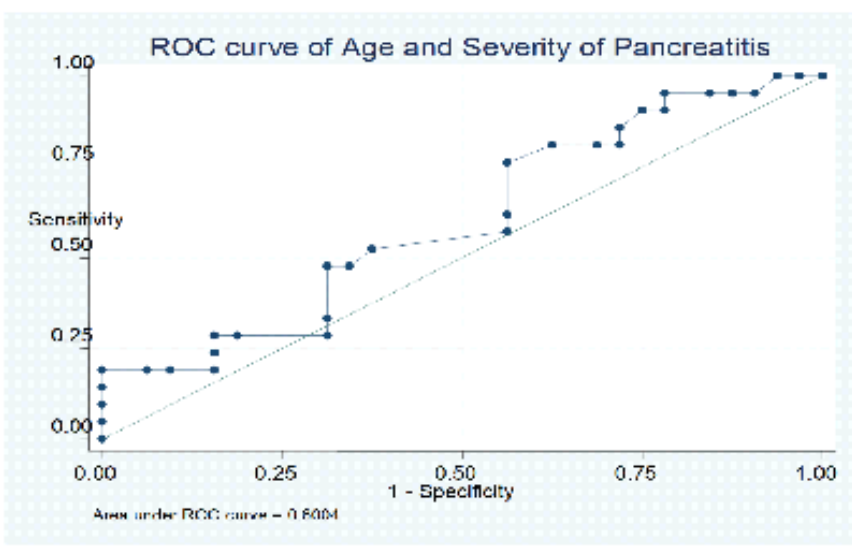

Figure 1: Accuracy of Age as predictors

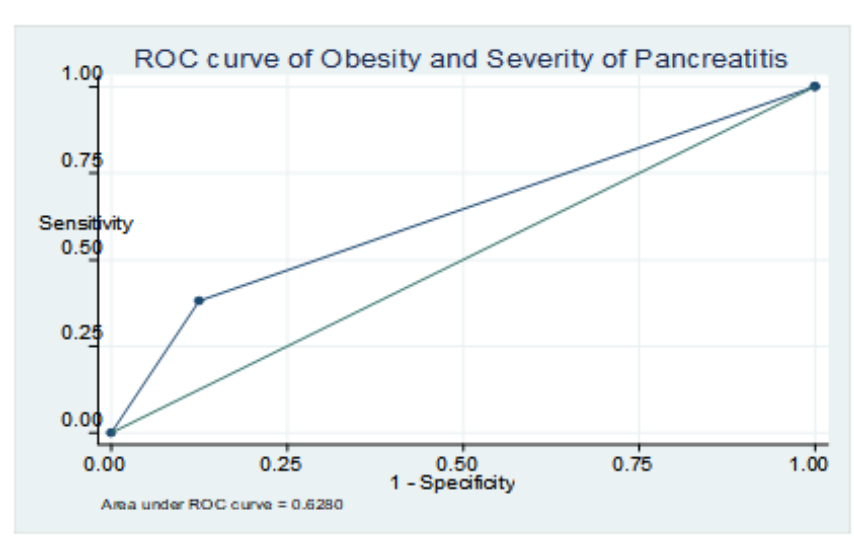

Figure 2: Obesity as a predictor

it was found that age was a poor predictor of the severity of acute pancreatitis.

[Figure 2] shows that the obesity of the patients was a significant indicator to discriminate or predict patients with mild or severe pancreatitis. With an AUROC (Area under ROC) of 0.6004 , it was found that age is a poor predictor of the severity of acute pancreatitis.

[Figure 3] shows that although bivariate analysis demonstrated that Organ failure at admission is more likely to reflect severe cases, it is found to be a poor predictor of severity in acute pancreatitis, when plotted on a ROC with an AUC of 0.6429.

[Figure 4] shows that the Ranson's score at 48 hours after admission of the patients in the study ranged from 0 to 6 with a mean (SD) of 1.68 (1.68) and a median of 1. It was higher in severe pancreatitis and a score of $>=3$ was significantly associated with severe pancreatitis by bivariate analysis. The area under the ROC for a Ranson's score of $>=3$ was 0.7783 for predicting the severity of pancreatitis. The sensitivity, 


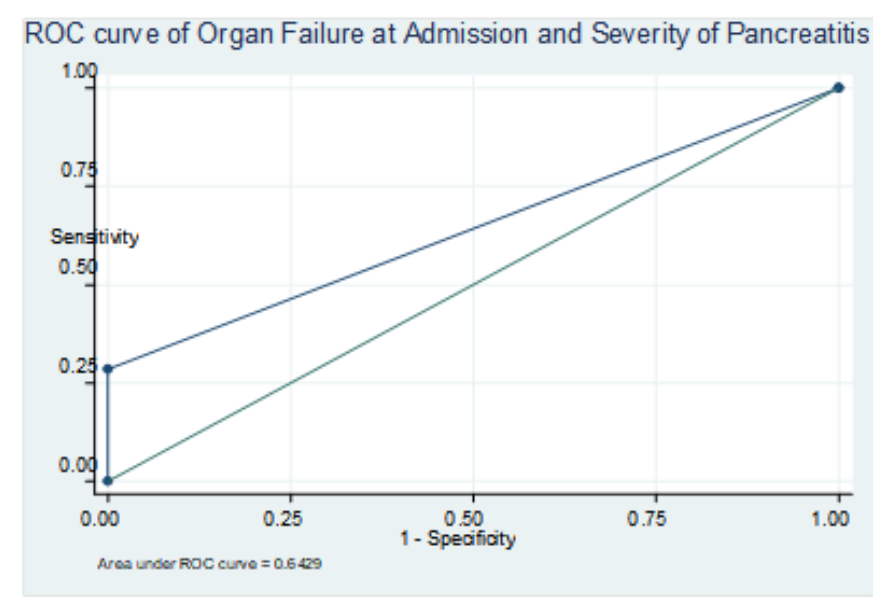

Figure 3: Organ failure at admission specificity, PPV, NPV and overall accuracy of Ranson's score $>=3$ in predicting the severity of pancreatitis was $62,94,87$, 79 , and $81 \%$, respectively.

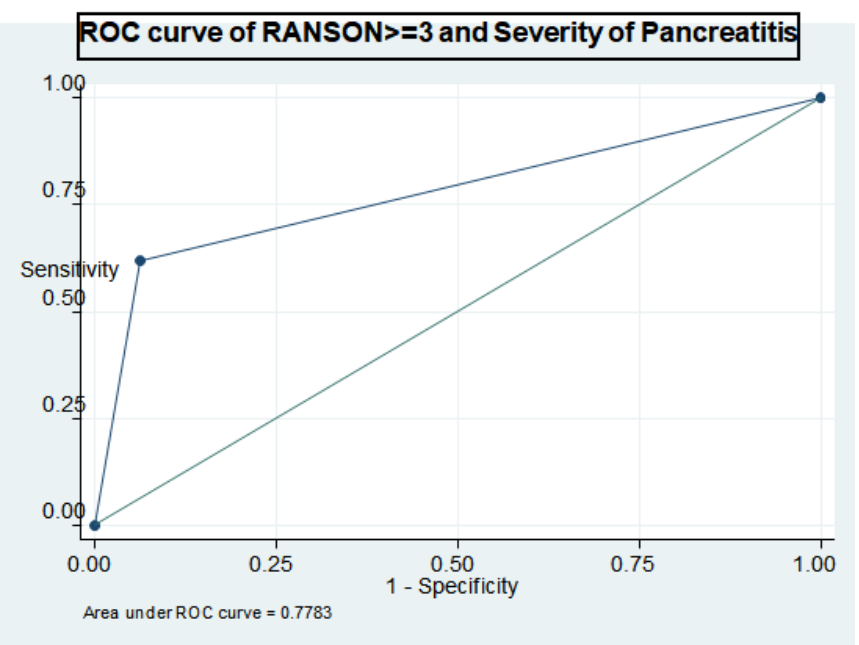

Figure 4: Ranson's scoring system

[Figure 5] shows that the Glasgow score of the patients in the study ranged from 0 to 5 with a mean (SD) of 1.38 (1.43) and a median of 1. It was higher in severe pancreatitis and a score of $>=3$ was significantly associated with patients with acute pancreatitis by bivariate analysis. The area under the ROC for a Glasgow score of $>=3$ was 0.7463 for predicting the severity of pancreatitis. The sensitivity, specificity, PPV, NPV and overall accuracy of Ranson's rating $>=3$ in predicting the severity of pancreatitis was $52,97,92,76$ and $79 \%$, respectively. With a cut off $>=2$, the AUROC was 0.82 , with a sensitivity of $76 \%$ and NPV of $83 \%$.

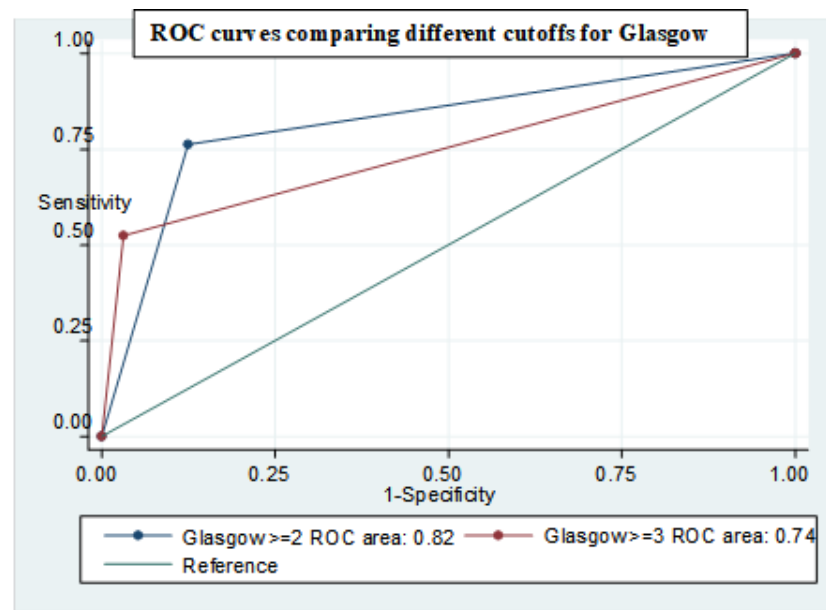

Figure 5: Glasgow scoring system

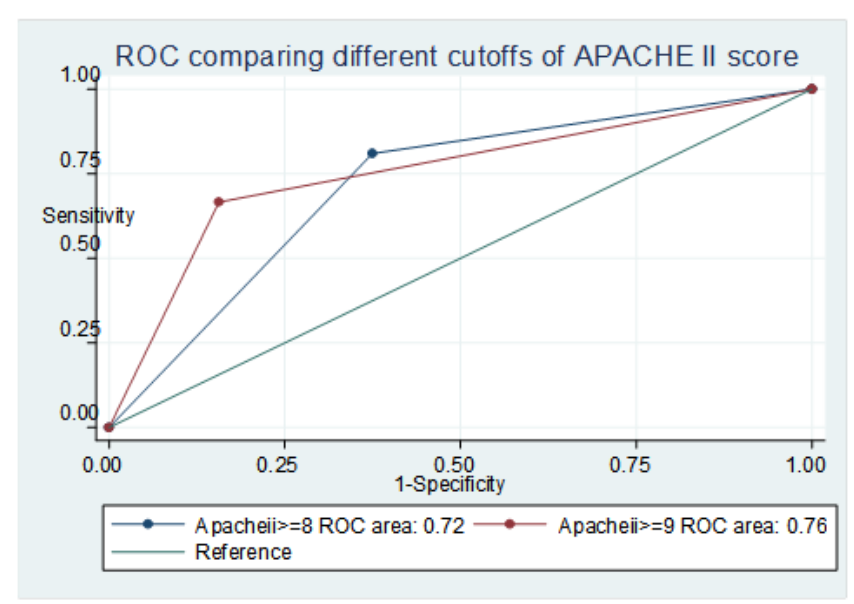

Figure 6: Apache-II scoring system

[Figure 6] shows that the total APACHE-II score of the patients in the study ranged from 4 to 20 with a mean (SD) of 8.28 (3.24) and a median of 8 . Bivariate analysis showed APACHE-II score to be higher in severe pancreatitis and a score of $>=8$ was significantly associated with acute pancreatitis. The AUROC for an APACHE-II score of $>=8$ was 0.7173 for predicting the severity of pancreatitis. The sensitivity, specificity, PPV, NPV and overall accuracy of APACHE-II score $>=8$ in predicting the severity of pancreatitis was $81,63,59,83$ and $70 \%$, respectively. With a cutoff of $>=9$, the AUROC was increased to 0.7552 and overall accuracy of $77 \%$.

[Figure 7] shows that the APACHE-O score of the patients in the study ranged from 4 to 22 with a mean (SD) of 8.79 (3.58) and a median of 8 . It higher in severe pancreatitis and 


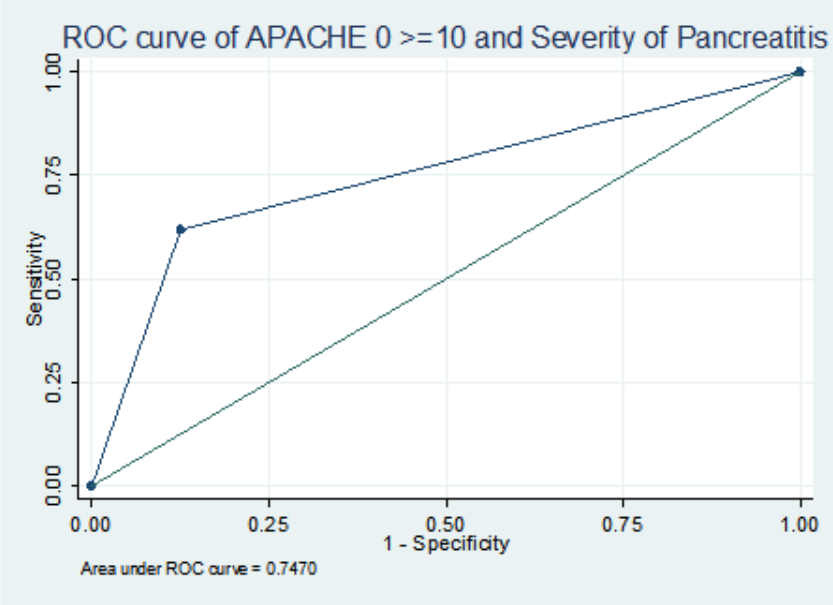

Figure 7: Apache-O scoring system

an APACHE-O score of $>=10$ was significantly associated with acute pancreatitis by bivariate analysis. The AUROC for an APACHE-O score of $>=10$ was 0.7068 for predicting the severity of pancreatitis. The sensitivity, specificity, PPV, NPV and overall accuracy of APACHE-O rating $>=10$ in predicting the severity of pancreatitis was $62,88,76,78$ and $77 \%$, respectively.

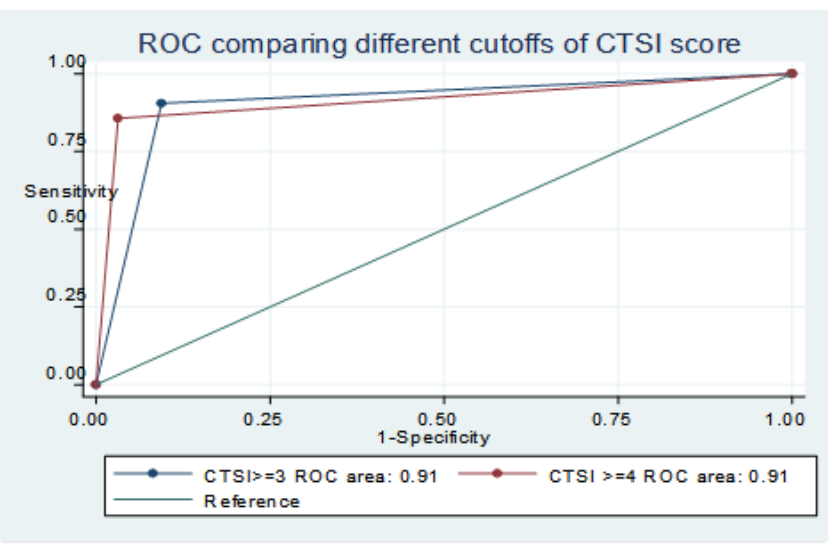

Figure 8: Balthazar's CTSI scoring system

[Figure 8] shows that the mean CTSI score in the study was 3.57 (SD 2.64) with a median of 2 and ranged from 1 to 10 . It was higher in severe pancreatitis and a CTSI score of $>=3$ was significantly associated with patients with acute pancreatitis by bivariate analysis. The area under the ROC for a CTSI score of $>=3$ was 0.9055 for predicting the severity of pancreatitis. The sensitivity, specificity, PPV, NPV and overall accuracy of CTSI score $>=3$ in predicting the severity of pancreatitis was $90,91,86,94$, and $91 \%$, respectively. With a cut of $>=4$, the
AUROC was marginally better with 0.9129 with sensitivity and overall accuracy of 95 and $92 \%$, respectively.

\begin{tabular}{|c|c|c|c|}
\hline Variable & AUC-ROC & STD error & $\begin{array}{ll}95 \% & \text { CI } \\
\text { Lower } & \\
\text { upper } & \end{array}$ \\
\hline CRP & 0.9754 & 0.0183 & $\begin{array}{l}0.93959 \\
1.00000\end{array}$ \\
\hline IL-6 & 0.9539 & 0.0292 & $\begin{array}{l}0.89660 \\
1.00000\end{array}$ \\
\hline PMN-E & 0.4301 & 0.0850 & $\begin{array}{l}0.26343 \\
0.59669\end{array}$ \\
\hline PCT & 0.8110 & 0.0562 & $\begin{array}{l}0.70090 \\
0.92112\end{array}$ \\
\hline
\end{tabular}

[Table 3] shows that among the biochemical markers, CRP was the best predictor of severity. Except for PMN-Elastase, all the biochemical markers are shown to be good predictors. PMN-Elastase was reasonable in discriminating diseased from ordinary but was a very poor predictor of the severity of pancreatitis. Overall, CRP was found to be the best predictor, followed by IL-6 and PCT.

[Table 4] shows that there was a significant difference in variables in mild and severe acute pancreatitis patients $(\mathrm{P}<$ $0.05)$.

\section{Discussion}

The pathophysiology of acute pancreatitis is usually considered in 3 phases. Phase I- Premature activation of trypsin within pancreatic acinar cells. Phase II- Intra pancreatic inflammation and phase III- Extra pancreatic inflammation. ${ }^{[7]}$ In some instances, the various pathways that contribute to increased intrapancreatic and extrapancreatic inflammation result in what is generally termed systemic inflammatory response syndrome (SIRS). ${ }^{[8]}$ In $20-30 \%$, it predisposes to multiple organ dysfunction and/or pancreatic necrosis. The factors that determine severity are not clearly understood but appear to involve a balance between pro-inflammatory and anti-inflammatory factors. ${ }^{[9]}$ This study was conducted to compare the multifactor vs independent marker in predicting the severity of acute pancreatitis.

In our study, there were 50 patients, of whom 45 were males and 5 were females. Cho et al, ${ }^{[10]}$ found that of 161 patients, $21(13 \%)$ were classified as severe AP, and $3(1.9 \%)$ died. Statistically significant cutoff values for prediction of severe AP were Ranson $\geq 3$, BISAP $\geq 2$, APACHE-II $\geq 8$, CTSI $\geq 3$, and CRP24 $\geq 21.4$. AUCs for Ranson, BISAP, APACHE-II, CTSI, and CRP 24 in predicting severe AP were 0.69 (95\%ci: 0.62-0.76), 0.74 (95\%ci: 0.66-0.80), 


\begin{tabular}{|c|c|c|c|}
\hline Variable & $\begin{array}{l}\text { Mild Pan- } \\
\text { creatitis } \\
(\mathrm{N}=32)\end{array}$ & $\begin{array}{l}\text { Severe } \\
\text { Pancreatitis } \\
(\mathrm{N}=18)\end{array}$ & P-value \\
\hline Age & $\begin{array}{l}34.72 \quad \pm \\
12.73\end{array}$ & $42 \pm 16.93$ & 0.08 \\
\hline $\begin{array}{l}\text { Obesity (n, } \\
\%)\end{array}$ & $4(12.50 \%)$ & $8(38.10 \%)$ & 0.05 \\
\hline RANSON & $0.84 \pm 0.95$ & $2.95 \pm 1.77$ & 0.0000 \\
\hline $\begin{array}{l}\text { RANSON } 3 \\
(\mathrm{n}, \%)\end{array}$ & $2(6.25)$ & $13(61.90)$ & $<0.001$ \\
\hline GLASGOW & $0.66 \pm 0.79$ & $2.48 \pm 1.50$ & 0.0000 \\
\hline GLASGOW & $1(3.13)$ & $11(52.38)$ & $<0.001$ \\
\hline $\begin{array}{l}\text { APACHE- } \\
\text { II }\end{array}$ & $6.94 \pm 2.09$ & $10.33 \pm 3.64$ & 0.0001 \\
\hline $\begin{array}{l}\text { APACHE- } \\
\text { II } \geq 8 \quad(\mathrm{n}, \\
\%)\end{array}$ & $12(37.50)$ & $17(80.95)$ & 0.002 \\
\hline $\begin{array}{l}\text { APACHE- } \\
\text { O }\end{array}$ & $7.34 \pm 2.10$ & $11 \pm 4.24$ & 0.0001 \\
\hline $\begin{array}{l}\text { APACHE- } \\
\mathrm{O} \geq 10\end{array}$ & $4(12.50)$ & $13(61.90)$ & $<0.001$ \\
\hline CTSI & $1.90 \pm 0.44$ & $6.15 \pm 2.54$ & 0.0000 \\
\hline $\begin{array}{l}\mathrm{CTSI} \geq 3 \quad(\mathrm{n}, \\
\%)\end{array}$ & $3(9.38)$ & $19(90.48)$ & $<0.001$ \\
\hline CRP & $44.35 \pm 53.04$ & $174.80 \pm 14.55$ & 0.0000 \\
\hline IL-6 & $129.63 \pm 319$. & $1166.76 \pm 818$ & 0.0000 \\
\hline PMN-E & $3.89 \pm 1087$ & $3.99 \pm 2.75$ & 0.88 \\
\hline PCT (n, \%) & $4(12.50)$ & $12(57.14)$ & $<0.001$ \\
\hline
\end{tabular}

0.78 (95\%ci: $0.70-0.84), 0.69$ (95\%ci: $0.61-0.76$ ), and 0.68 (95\%ci: 0.57-0.78), respectively. APACHE-II demonstrated the highest accuracy for the prediction of severe AP. However, no statistically significant pairwise differences were observed between APACHE-II and the other scoring systems, including CRP.

In patients with acute pancreatitis, early gradation of disease severity is essential to provide optimum supportive care in intensive units, high dependency units or wards, especially with limited health-care resources as well as to plan for appropriate interventional procedures viz ERCP in biliary pancreatitis. About $50 \%$ of deaths occur within 1 week of the attack, mostly from multi-organ dysfunction syndrome. Various single markers have been tested to replace the multifactor systems, of which the most successful has been Creactive protein.

We found that there were 12 (22.64\%) obese patients in this study. We found that age of the patients was a significant indicator to discriminate or predict patients with mild or severe pancreatitis. With an AUROC of 0.6004 , it was found that age was a poor predictor of the severity of acute pancreatitis. Obesity of the patients was a significant indicator to discriminate or predict patients with mild or severe pancreatitis. Organ failure at admission is more likely to reflect severe cases, it is found to be a poor predictor of severity in acute pancreatitis when plotted on a ROC with an AUC of 0.6429 .

Ranson's score at 48 hours after admission of the patients in the study ranged from 0 to 6 with a mean (SD) of 1.68 (1.68) and a median of 1 . It was higher in severe pancreatitis and a score of $>=3$ was significantly associated with acute pancreatitis by bivariate analysis.

Glasgow score of the patients in the study ranged from 0 to 5 with a mean (SD) of $1.38(1.43)$ and a median of 1 . It was higher in severe pancreatitis and a score of $>=3$ was significantly associated with patients with acute pancreatitis by bivariate analysis. The total APACHE-II score of the patients in the study ranged from 4 to 20 with a mean (SD) of 8.28 (3.24) and a median of 8. Bivariate analysis showed APACHEII score to be higher in severe pancreatitis and a score of $>=8$ was significantly associated with acute pancreatitis.

The APACHE-O score of the patients in the study ranged from 4 to 22 with a mean (SD) of 8.79 (3.58) and a median of 8. It higher in severe pancreatitis and an APACHE-O score of $>=10$ was significantly associated with acute pancreatitis by bivariate analysis. The mean CTSI score in the study was 3.57 (SD 2.64) with a median of 2 and ranged from 1 to 10. It was higher in severe pancreatitis and a CTSI score of $>=3$ was significantly associated with patients with acute pancreatitis by bivariate analysis. The biochemical markers, CRP was the best predictor of severity. Except for PMNElastase, all the biochemical markers are shown to be good predictors. PMN-Elastase was reasonable in discriminating diseased from ordinary but was a very poor predictor of the severity of pancreatitis. Overall, CRP was found to be the best predictor, followed by IL- 6 and PCT. There was a significant difference in variables in mild and severe acute pancreatitis patients $(\mathrm{P}<0.05)$.

It is hard to identify severe cases earlier than 2-3 days of symptom onset, by which time the network of pathophysiological mechanisms leading to multi-organ dysfunction syndrome is established. An ideal prognostic system would be based on a single test and have a high negative predictive value and should also be universally available, reproducible and nonexpensive. Existing methods rely on clinical-biochemical multifactor scoring systems, some of which involve cumbersome calculation. ${ }^{[11]}$ 


\section{Conclusion}

The authors found that overall, CRP was the best predictor, followed by IL-6, CTSI score, PCT, Glasgow, Ranson's and APACHE-II. PMN-Elastase, Age, obesity and organ failure at admission are poor predictors of severity of acute pancreatitis.

\section{References}

1. Ranson JHC, Pasternack BS. Statistical methods for quantifying the severity of clinical acute pancreatitis. J Surg Res. 1977;22(2):79-91. Available from: https://dx.doi.org/10.1016/ 0022-4804(77)90045-2. doi:10.1016/0022-4804(77)90045-2.

2. Imrie CW, Benjamin IS, Ferguson JC, McKay AJ, Mackenzie I, O'Neill J, et al. A single-centre double-blind trial of Trasylol therapy in primary acute pancreatitis. Br J Surg. 1978;65(5):337-341. Available from: https://dx.doi.org/10. 1002/bjs.1800650514. doi:10.1002/bjs.1800650514.

3. McKay CJ, Evans S, Sinclair M, Carter CR, Imrie CW. High early mortality rate from acute pancreatitis in Scotland, 1984-1995. Br J Surg. 1999;86(10):1302-1305. Available from: https://dx.doi.org/10.1046/j.1365-2168.1999. 01246.x. doi:10.1046/j.1365-2168.1999.01246.x.

4. Blamey SL, Imrie CW, O'Neill J, Gilmour WH, Carter DC. Prognostic factors in acute pancreatitis. Gut. 1984;25(12):1340-1346. Available from: https://dx.doi.org/10. 1136/gut.25.12.1340. doi:10.1136/gut.25.12.1340.

5. Knaus WA, Draper EA, Wagner DP, Zimmerman JE. APACHE II-A Severity of Disease Classification System. Crit Care Med. 1986;14(8):755-755. Available from: https://dx.doi.org/10.1097/00003246-198608000-00028. doi:10.1097/00003246-198608000-00028.

6. Williams M, Simms HH. Prognostic usefulness of scoring systems in critically ill patients with severe acute pancreatitis. Crit Care Med. 1999;27(5):901-907. Available from: https://dx.doi.org/10.1097/00003246-199905000-
00023. doi:10.1097/00003246-199905000-00023.

7. Ryu JK. Evaluation of severity in acute pancreatitis. Korean J Gastroenterol. 2009;54:205-211.

8. Müller CA, Uhl W, Printzen G, Gloor B, Bischofberger H, Tcholakov $\mathrm{O}$, et al. Role of procalcitonin and granulocyte colony-stimulating factor in the early prediction of infected necrosis in severe acute pancreatitis. Gut. 2000;46:233-238.

9. Puolakkainen P, Valtonen V, Paananen A, Schroder T. Creactive protein (CRP) and serum phospholipase A2 in the assessment of the severity of acute pancreatitis. Gut. 1987;28(6):764-771. Available from: https://dx.doi.org/10. 1136/gut.28.6.764. doi:10.1136/gut.28.6.764.

10. Cho JH, Kim TN, Chung HH, Kim KH. Comparison of scoring systems in predicting the severity of acute pancreatitis. World J Gastroenterol. 2015;21:2387-2394. doi:10.3748/wjg.v21.i8.2387.

11. Leung TK, Lee CM, Lin SY, Chen HC, Wang HJ, Shen LK, et al. Balthazar computed tomography severity index is superior to Ranson criteria and APACHE II scoring system in predicting acute pancreatitis outcome. World J Gastroenterol. 2005;11:6049-6052. doi:10.3748/wjg.v11.i38.6049.

Copyright: (C) the author(s), 2020. It is an open-access article distributed under the terms of the Creative Commons Attribution License (CC BY 4.0), which permits authors to retain ownership of the copyright for their content, and allow anyone to download, reuse, reprint, modify, distribute and/or copy the content as long as the original authors and source are cited.

How to cite this article: Bada VC. Comparison of Multifactor Vs Independent Marker in Predicting Severity of Acute Pancreatitis. Acad. J Surg. 2020;3(1):136-141.

DOI: dx.doi.org/10.47008/ajs/2020.3.1.30

Source of Support: Nil, Conflict of Interest: None declared. 